\title{
Skills, innovation, and growth: An agent-based policy analysis
}

\author{
H. Dawid, S. Gemkow ${ }^{\dagger}$, P. Harting ${ }^{\dagger}$, K. Kabus ${ }^{\dagger}$, \\ M. Neugart ${ }^{\dagger}$ and K. Wersching ${ }^{\dagger}$
}

\begin{abstract}
We develop an agent-based macroeconomic model featuring a distinct geographical dimension and heterogeneous workers with respect to skill types. The model, which will become part of a larger simulation platform for European policymaking (EURACE), allows us to conduct ex-ante evaluations of a wide range of public policy measures and their interaction. In particular, we study the growth and labor market effects of various policy types that promote workers' general skill levels. Using a calibrated model it is examined in how far effects differ if spending is uniformly spread over all regions in the economy or focused in one particular region. We find that the geographic distribution of policy measures significantly affects the effects of the policy even if total spending is kept constant. Focussing training efforts in one region is the worst policy outcome while spreading funds equally across regions generates a larger output in the long-run but not in the short-run.
\end{abstract}

\section{Introduction}

Normative research in economics has traditionally been, and to a large degree still is based on the development and analysis of highly stylized, analytically tractable models. In particular for macroeconomic issues the models used for policy analysis are typically dynamic general equilibrium models that have been calibrated using empirical data. However, numerous restrictive assumptions underly most mainstream analytical models (e.g. homogeneity of

*Department of Business Administration and Economics, Bielefeld University, Germany

${ }^{\dagger}$ School of Economics and Management, Free University of Bozen-Bolzano, Italy 
individuals, perfect rationality, rational expectations, perfect ex-ante coordination in an equilibrium) and so far there exists almost no general theoretical basis that allows to judge how far findings, obtained under these simplifying assumptions, carry over to scenarios where agents are heterogeneous or out of equilibrium (see e.g. Kirman (1992)). On the other hand, recent developments in computer technology and software engineering have made large scale simulations an increasingly powerful and attractive new approach for understanding the characteristics of economic systems and for deriving economic policy recommendations. In particular, by explicitly modelling the decentralized interaction of heterogeneous economic agents in systems like markets or organizations, agent-based computational economics (ACE) attempts to transcend the limitations of traditional models.

The ACE modeling approach is not only well suited for explicit consideration of heterogeneity among economic agents, but also allows for a wide range of assumptions concerning the rules that determine the behavior of individual economic agents. Existing ACE models typically consider adaptive individuals who learn how to behave and react based on their own experience and on the available information ${ }^{1}$. Models of this kind have been developed in many areas of economics. Among others, they have been used to study the emergence of trading behavior on goods-markets and on financial markets, bidding behavior in auctions, numerous issues concerning innovation and industry evolution or the emergence of cooperative behavior in economic systems. Surveys over agent-based research in these areas can be found in Tesfatsion and Judd (2006).

A major advantage of the agent-based simulation approach is that the modeler can easily study the effects of changes in the economic framework on various aspects of the resulting economic dynamics and long run outcome. Accordingly, agent-based models have a large potential as a tool to evaluate the impact of public policy measures or of changes in market design. Whereas early ACE-work has been mainly of descriptive character, in recent years there have been a number of projects where agent-based models have been employed to (normatively) address actual market design and public policy questions (see e.g. Dawid and Fagiolo (2007)). The considered policy issues relate to electricity markets (e.g. Bunn and Oliveira (2001), Nicolaisen et al. (2001)), labor market design (e.g. Neugart (2007)), auction design (e.g. Phelps et al. (2002)), patent policy (e.g. Malerba et al. (2001)) or agricultural policy (e.g. Happe et al. (2007)). This list of issues demonstrates that so far the focus of agent-based normative analysis has

\footnotetext{
${ }^{1}$ Work by Cyert and March (1963), Simon (1978, 1983) or Nelson and Winter (1982) has been influential for the development of this line of research.
} 
been on questions of market-design and have typically involved partial models of particular markets. Agent-based work in the area of macroeconomic modelling is however sparse. Closed macroeconomic models using an ACE approach have been provided for example by Chiaromonte and Dosi (1993), Silverberg and Verspagen (1993), Delli Gatti et al. (2005), and Dosi et al. (2006). But the focus of this work is on demonstrating that the models are able to simultaneously reproduce a large number of empirically observable stylized facts rather than on the evaluation of economic policy measures. In a recent paper Gintis (2006) employs an agent-based macroeconomic model to study the dynamics of multi-sector Walrasian general equilibrium models. An interesting closed agent-based model with particular focus on the reproduction of several stylized facts in the labor market has been provided by Fagiolo et al. (2004).

The purpose of this paper is to demonstrate that agent-based closed macroeconomic models are not only well suited to reproduce empirically observed regularities but can be usefully applied to evaluate economic policy measures in a way that is infeasible for representative agent models. In particular, we focus on an area that has recently received strong attention by policy makers in industrialized countries, namely the question what kind of economic policy measures are best suited to facilitate innovation and diffusion of new technologies and productivity increase. Dealing with this question requires to shed more light on the interplay of processes leading to the generation of new technologies and the ability of firms to adopt such new technologies. In order to efficiently use new technologies the workforce of the industrial firms has to be able to build up the required level of specific skills and the ability to do so depends on the general skills levels of the employees. There is strong empirical evidence that the skill distribution in the workforce has substantial influence on the speed of technological change, the employment and wage dynamics and growth in an economy (e.g. Bassanini and Scarpetta (2002), Bassanini (2004)). For example empirical studies initiated by the OECD economics division (see e.g. OECD (2000)) indicate that the lack of the new forces of economic growth and relatively low-skill levels in the labor force have negative effects on employment in Europe. In particular, the U.S. took the lead since the 1990's in creating new knowledge, human capital and innovations accompanied by entrepreneurship and new start-up firms which gave rise to higher employment. Therefore, policies aiming at a change in the local skill distribution play an important role in fostering technological change and growth. Any model designed to evaluate such policies, and more generally any technology and growth policy measures, miss a crucial aspect if they do not incorporate the dynamics of skill and knowledge distribution in the workforce. 
To be more concrete, we can address questions concerning the vivid policy debate whether strengthening general or specific skills is more likely to enhance growth and employment. For example in Germany the dualapprenticeship system is notoriously questioned by firms, unions and policymakers with regard to its provision of general skills. A common complaint concerning university education in Germany is the high level of the abstraction of the curriculum which provides only limited specific skills. Currently there are no theoretical models that allow to analyze these issues based on thorough macroeconomic analysis. In particular standard analyses conducting cost/benefit analysis of dual apprenticeship systems are based on partial static analysis (Mühlemann et al. (2007)). Obviously, in a world with fast changing technological frontiers this does not fully take into account the full benefits of workers' general skills. Gaining better insights into the effects of increases in both types of skills should have impact on the balance of public spending between primary and secondary education, tertiary eduction, life-long learning measures and dual apprenticeships.

An additional aspect in the policy debate about the optimal design of educational policies fostering innovation and growth is the question in how far such policies should depend on the technological distance of the firms in the economy from the technological frontier. Claims have been made that in regions far from the frontier the main focus should be on primary and secondary education improving the lower end of the skill distribution, whereas regions at the frontier profit more from higher investments in the tertiary sector (see Aghion (2007)). From a dynamic perspective this raises the questions in how far the effectiveness of different policy approaches depends on the speed at which the technological frontier is moving, which itself is influenced by measures in the area of innovation and technology policy. Such measures include among others direct funding of basic and applied research, financial incentives for R\&D efforts by firms, providing infrastructure and incentives for $\mathrm{R} \& \mathrm{D}$ cooperation and intensive knowledge exchange between research institutions and firms.

In that respect spatial aspects also play a crucial role. First, in many industrialized countries there are strong regional differences in the skill and knowledge distribution where high-skill employees are strongly concentrated in a few areas. Second, geographic proximity between firms has a crucial impact on the intensity of technological spillovers between them. Both theoretical and empirical studies of innovative activities have demonstrated the importance of technological spillovers for industry development (see e.g. the surveys by Audretsch and Feldman (2004) and Döring and Schnellenbach (2006)). Whereas the main channels through which technological information flows between firms depends heavily on the type of industry considered 
(see e.g. Geroski (1995)), a considerable role in establishing technological spillovers is typically assigned to direct communication and the flow of skilled and well informed employees. Therefore, the interaction of firms and employees on the (local) labor market is of great importance for the size of technological spillovers and, hence, for innovative activities. Taking into account regional differences and the existence of (local) knowledge flows between firms the question arises to what extent economic policy measures should be regionally differentiated. In particular, it has to be explored how the effects of certain skill enhancing policy measures differ when applied in regions with different characteristics. Furthermore, the right allocation of policy measures among all regions in the economy is a difficult problem. Should the activity be centered on the strongest or on the weakest region or should all measures be uniformly distributed across regions? The type of insights about crucial medium and long run effects we can generate using our model allows policy makers to get a broader picture of the implications of different allocations of public funds and therefore provides an important contribution to policy advice.

In previous years the main approach for the analysis of spatial aspects of economic activity has been the use of 'New Economic Geography' (NEG) models introduced by Krugman (1991). These models rely on a number of simplifying assumptions like the restriction to two regions or equidistant multi-regions (see Bosker et al. (2007)), the absence of strategic behavior of firms or the lack of considerations of institutional aspects. These restrictions, which are mainly due to the desire to keep the models tractable, have been acknowledged by NEG researchers. For example in their survey of recent NEG work Ottaviano and Thisse (2004) point out that 'By their very nature, such models are unable to, explain the rich and complex hierarchy that characterizes the space-economy. ... Therefore, one major step on the research agenda is the study of a multi-regional system whose aim is to understand why some regions are more successful than others.' [p. 2603]. Also some limitations of NEG concerning the study of spatial dynamics on the labor market is acknowledged in Ottaviano and Thisse (2004): 'Another fundamental question is related to the fact that local labor markets are modeled in a very simple way in NEG: operating profits are used to pay skilled workers. In particular, these models do not help understand why unemployment persists in areas included in or adjacent to prosperous regions.'[p. 2604]. These restrictions of NEG models imply that they are not well suited to address the (spatial) policy questions discussed above. Actually, the work using an NEG approach for normative analysis of economic policy is limited. An indicator in that respect is that the recently published Handbook of Regional and Urban Economics (Henderson and Thisse (2004)), where a lot of attention is dedicated to NEG, 
does not include any chapters discussing economic policy.

Based on the obvious restrictions imposed by analytical tractability on the spatial analysis of the interplay of technological change and properties of the labor force, the need for an alternative approach for the evaluation of different combinations of economic policy measures seems particularly strong. Agent-based models are well suited to address this need ${ }^{2}$. In this paper we sketch an agent-based model that is as simple as possible but still apt to address some of the important policy questions raised above. It is a closed model consisting of a consumption goods and an investment (or capital) goods sector. Households and firms are distributed across regions. Some markets (consumption goods) are assumed to be local, some are global (investment goods) and workers might commute for work to employers in neighboring regions at certain costs. Investment goods are supplied at an exogenously given price. The inputs for consumption good production are investment goods and labor. Process innovation improves the quality of investment goods leading to higher productivity of capital in the consumption good production. The labor market hosts workers of different types. They are differentiated along their general as well as their specific skill level. A crucial assumption is that sufficient specific skills of workers are needed to exploit the full potential of the advanced technological level of investment goods like production machines. Put formally, there is complementarity between the average quality of the investment goods of a firm and the average level of specific skills of its employees with respect to the productivity in consumption good production. Empirical evidence for such complementarity can for example be found in Griffith et al. (2004). Workers of higher general skills adapt faster in terms of their specific skills needed to produce consumption goods by the use of investment goods of a particular quality. General skills affecting the adaptation of specific skills will allow us to study the effects of government policies improving the general skill level of the workforce on employment and growth.

A general problem of agent-based models, that attempt to avoid the (overly) strong assumptions about information and rationality of individuals underlying equilibrium analysis, is the appropriate design of decision rules that govern the behavior of individual agents. Deviation from the intertemporal (constrained) maximization paradigm opens many degrees of freedom with respect to the type of behavioral rules used and the way behavior is adapted over time. However, as far as firm behavior is concerned for many operational decisions, like pricing, production and inventory choice or market

\footnotetext{
${ }^{2}$ See Dawid and Wersching (2006) or Wersching (2007) for agent-based analyses of spatial aspects of industry dynamics.
} 
selection decisions, standard decision rules and heuristics have been developed that are well documented in the relevant business and operations management literature. Our 'philosophy' in terms of modelling firm behavior is to implement relatively simple decision rules that match standard procedures of real world firms as described in the corresponding management literature. In a similar spirit the decisions of consumers, like the allocation of the available budget between consumption and savings, is modelled according to simple empirically founded rules from the literature.

Apart from the fact that behavioral rules of individual agents in the model have to be in accordance with stylized representations of standard decision rules employed by their real-world representatives, it is also important to critically examine the plausibility of the used parameterization and the qualitative patterns of simulation results. Concerning parametrization it is important to employ empirical insights with respect to all model parameters where direct evidence exists. We follow this approach and calibrate the parameters in our model using estimates from different streams of relevant literature. Concerning model evaluation a widely used approach in recent work in agent-based economics is to compare simulation outcomes with 'stylized facts' that have been established using real world data. This kind of comparison is supposed to restrict the range of model parameters and to improve the confidence that the model captures crucial aspects of interactions in the sectors considered in the model, see Windrum et al. (2007) for a discussion of approaches to validate agent-based simulation models. Our model reproduces several stylized facts, but due to space constraints we only show a few variables and in particular with respect to skill specific labor outcomes we can only sketch our findings. We rather focus on the presentation of the economic logic exhibited by our policy experiments.

The strong empirical footing of the developed agent-based model is not only of great importance for purely scientific reasons but is also crucial to establish trust of actual policy makers in the results of the policy analysis and the policy recommendations generated by the model. The model and preliminary analysis presented in this paper is part of the EU-funded project (EURACE) 'An Agent-based Software Platform for European Economic Policy Design with Heterogeneous Interacting Agents: New Insights from a Bottom-Up Approach to Economic Modeling and Simulation?3. The main purpose of the project is to develop a unified agent-based macroeconomic simulation platform that can be used to inform policy makers about expected effects of (combinations of) various economic policy measures. Our

\footnotetext{
${ }^{3}$ See http://www.eurace.org or http://www.wiwi.uni-bielefeld.de/ 2dawid/eurace/ for more information about the EURACE project.
} 
focus here is on industrial and labor-market issues, but the goal of the entire project is to cover all main areas of economic policy. Therefore, the model presented in this paper is only a part of the full EURACE model, in particular explicit models of financial and credit markets are not included here, although they are important parts of the EURACE model.

We proceed as follows. The main features of the simulation model are described in section 2 . In section 3 we briefly present key features of simulation results generated by the model and then discuss the potential of the framework to evaluate different types of policy measures that aim at faster economic growth. The potential of the model for policy analysis is then illustrated by comparing the effects of different degrees of spatial concentration of policy measures. We conclude in section 4 with a brief discussion and pointers to future work.

\section{The model}

\subsection{General features}

Our model consists of a capital good, a consumption good, and a labor market. 4 Capital goods are provided with infinite supply at exogenously given prices. The quality of the capital good improves over time where technological change is driven by a stochastic (innovation) process. Firms in the consumption goods sector use capital goods combined with labor input to produce consumption goods. The labor market is populated with workers that have a finite number of general skill levels and acquire specific skills onthe-job which they need to fully exploit the technological advantages of the capital employed in the production process. Consumption goods are sold at malls. Malls are not treated as profit-oriented enterprizes but simply as local market platforms where firms store and offer their products and consumers come to buy goods at posted prices.

Thus, two types of active agents and two types of passive agents (in the sense that this type of agent does not take any decisions) are present in the model. Each type of active agent has several 'roles' corresponding to its activities in the different markets. Table 1 summarizes these roles.

The economy consists of $R=2$ regions and each agent is located in one of these regions. Some actions occur locally, such as the agents' consumption, others occur globally including the sale of the investment good or labor

\footnotetext{
${ }^{4}$ In the fully fledged EURACE model, a financial and a credit market will be added, and an exogenous energy market will constitute a proxy for the link to the 'rest-of-the-world' by affecting the production costs in the capital goods market.
} 


\begin{tabular}{|c|c|c|}
\hline \multirow[t]{2}{*}{$\begin{array}{l}\text { Active } \\
\text { Agents }\end{array}$} & Households & $\begin{array}{l}\text { - Consumption Goods Market: Role of Buyer } \\
\text { - Labor Market: Role of Worker }\end{array}$ \\
\hline & $\begin{array}{l}\text { Consumption } \\
\text { Goods } \\
\text { Producer }\end{array}$ & $\begin{array}{l}\text { - Investment Goods Market: Role of Buyer } \\
\text { - Consumption Goods Market: Role of Seller } \\
\text { - Labor Market: Role of Employer }\end{array}$ \\
\hline \multirow[t]{2}{*}{$\begin{array}{r}\text { Passive } \\
\text { Agents }\end{array}$} & Malls & $\begin{array}{l}\text { - Consumption Goods Market: Information } \\
\text { Transfer between Producers and Households }\end{array}$ \\
\hline & $\begin{array}{l}\text { Capital } \\
\text { Goods } \\
\text { Producer }\end{array}$ & - Investment Goods Market: Role of Seller \\
\hline
\end{tabular}

Table 1: Agents and their role in the model.

supply.

Generally, the minimal unit of time is a day, however almost all the interactions and decisions are repeated on a monthly basis. ${ }^{5}$ Therefore, whenever we refer to one time-period by default we mean one month. Some decisions in the consumption goods market are taken on a weekly basis and we will explicitly point out this fact in the text.

\subsection{Investment goods market}

There exists a single type of technology for investment goods. The investment good is offered with infinite supply. The quality of the investment good $q_{t}^{i n v}$ increases over time due to a stochastic process. Every period the quality is increased with probability $\gamma^{i n v} \in(0,1)$ where with probability $\left(1-\gamma^{i n v}\right)$ there is no change of quality. In case of an increase the quality of the offered good changes by a fixed percentage $\Delta q^{i n v}$.

The price of the investment good $p^{i n v}>0$ is assumed to be linked to the level of quality, so that a rise of quality leads to a proportional increase of $p^{i n v}$. Although capital goods producers are not modelled as active agents the amounts paid for investment goods are channeled back into the economy. Revenues accruing with the investment good producer are distributed in equal shares among all households in order to close the model. Put differently, it is assumed that all households own equal shares in the fictitious capital goods producer.

\footnotetext{
${ }^{5}$ In the model each week consists of 5 days and each month of 4 weeks. Accordingly, each year has 240 days.
} 


\subsection{Consumption good producer}

\subsubsection{Quantity choice}

Every consumption goods producer keeps a stock of its products at every regional mall. For simplicity it is assumed that all producers offer their products in both regions. A producer checks once every period whether any of the stocks it keeps at different malls have to be refilled. To that end the firm receives messages from all the malls it serves reporting the current stock level. Taking this information into account, the firm $i$ has to decide whether and on what scale it restocks the supply. According to our approach of using standard managerial methods wherever it is applicable, we employ a standard inventory rule for managing the stock holding. For reasons of simplicity we ignore setup costs that arise for each delivery to a mall. We denote by $C_{i, r}^{i n v}$ costs of holding one unit of the good in the inventory for one period and by $\tilde{\Phi}_{i, r, t}(D):[0, \infty) \mapsto[0,1]$ the estimated distribution function of the demand for the good of firm $i$ at the mall in region $r$, where the estimation is based on demands reported by the mall in the previous $T$ periods. Furthermore, $S L_{i, r, t}$ is the level of the stock of firm $i$ at the mall in region $r$ at the day in period $t$ when the stock is checked. Then, standard results from inventory theory suggest that the firm should choose its desired replenishment quantity for region $r$ according to the following simple rule (see Hillier and Lieberman (1986)):

$$
\tilde{D}_{i, r, t}= \begin{cases}0 & S L_{i, r, t} \geq Y_{i, r, t} \\ Y_{i, r, t}-S L_{i, r, t} & S L_{i, r, t}<Y_{i, r, t},\end{cases}
$$

where $Y_{i, r, t}$ is the smallest value $Y \geq 0$ that satisfies

$$
\tilde{\Phi}_{i, r, t}(Y) \geq \frac{p_{i, r, t}-(1-\rho) \bar{c}_{i, t-1}}{p_{i, r, t}+C_{i, r}^{i n v}} .
$$

Here $\bar{c}_{i, t-1}$ denotes unit costs of production for firm $i$ in the previous period, $p_{i, r, t}$ the prices of the consumption good, and $\rho$ the discount factor. The sum of the orders received by all malls becomes

$$
\tilde{D}_{i, t}=\sum_{r=1}^{R} \tilde{D}_{i, r, t} .
$$

To avoid excessive oscillations of the quantities $\tilde{Q}_{i, t}$ that the firm desires to produce in period $t$, the time-series of total quantities required by the different malls $\left(\tilde{D}_{i, t}\right)$ is smoothed. On this account, the consumption goods 
producer shows some inertia in adapting the actual production quantity to the quantity requested by the malls. In particular, we have

$$
\tilde{Q}_{i, t}=\xi \cdot \tilde{D}_{i, t}+(1-\xi) \cdot \frac{1}{T} \cdot \sum_{k=t-T}^{t-1} Q_{i, k} .
$$

As discussed in more detail below, the realized production volume $Q_{i, t}$ can deviate from the planned output $\tilde{Q}_{i, t}$ due to rationing on the factor markets. The quantities actually delivered to the malls, $D_{i, r, t}$, are adjusted proportional to the intended quantities $\tilde{D}_{i, r, t}$, so that

$$
D_{i, r, t}=\frac{\tilde{D}_{i, r, t}}{\sum_{r^{\prime}=1}^{R} \tilde{D}_{i, r^{\prime}, t}} \cdot Q_{i, t} .
$$

Production times of consumption goods are not explicitly taken into account and the produced quantities are delivered on the same day when production takes place. The local stock levels at the malls are updated accordingly.

\subsubsection{Factor demand}

Consumption good producers, denoted by $i$, need physical capital and labor to produce the consumption goods. The accumulation of physical capital by a consumption good producer follows

$$
K_{i, t+1}=(1-\delta) K_{i, t}+I_{i, t}
$$

where $K_{i}(0)=0$ and $I_{i, t}>0$ is the gross investment.

Every worker $w$ has a level of general skills $b_{w}^{\text {gen }} \in\left\{1, \ldots, b_{\text {max }}^{\text {gen }}\right\}$ and a level of specific skills $b_{w, t}$. The specific skills of worker $w$ indicate how efficiently the corresponding technology is exploited by the individual worker. Building up those specific skills depends on collecting experience by using the technology in the production process. There is vast empirical evidence for such adjustment processes (see e.g. Argote and Epple (1990)). The shape of the evolution of productivity follows a concave curve, the so-called learning curve, when the organizational productivity is recorded after implementing a new production method or introducing a new good. Concavity in this context means that the productivity rises with proceeding use of the production method or production of the new good, but this increase emerges at a decreasing rate. We transfer this pattern of organizational learning on the individual level and assume that the development of individual productivity follows a learning curve. The specific skills are updated once in each production cycle of one month. Further, we assume that updating takes place at the end of the cycle. 
A crucial assumption is the positive relationship between the general skills $b_{w}^{g e n}$ of a worker and his ability to utilize his experiences. Building up worker's technology specific skills depends on a worker's level of general skills, i.e. his education and the other abilities which are not directly linked to the particular technology. Taking the relevance of the general skill level into account the specific skills of a worker $w$ for technology $j$ is assumed to evolve according to

$$
b_{w, t+1}=b_{w, t}+\chi\left(b_{w}^{g e n}\right) \cdot\left(A_{i, t}-b_{w, t}\right),
$$

where we denote with $A_{i, t}$ the average quality of the capital stock. The function $\chi$ is increasing in the general skill level of the worker. Note that this formulation captures the fact that in the absence of technology improvements marginal learning curve effects per time unit decrease as experience is accumulated and the specific skills of the worker approaches the current technological frontier.

The production technology in the consumption goods sector is represented by a Cobb-Douglas type production function with complementarities between the quality of the investment good and the specific skills of employees for using that type of technology. Factor productivity is determined by the minimum of the average quality of physical capital and the average level of relevant specific skills of the workers. Capital and labor input is substitutable with a constant elasticity and we assume constant returns to scale. Accordingly, output for a consumption goods producer is given by

$$
Q_{i, t}=\min \left[B_{i, t}, A_{i, t}\right] \times L_{i, t}^{\alpha} K_{i, t}^{\beta},
$$

where $B_{i, t}$ denotes the average specific skill level in firms and $\alpha+\beta=1$.

Firms aim to realize a capital to labor ratio according to the standard rule for CES production functions. That is a ratio of quantity to price of the two factors proportional to the corresponding intensity parameter. Accordingly,

$$
\frac{\tilde{K}_{i, t}}{p^{i n v}} / \frac{\tilde{L}_{i, t}}{w_{t}^{e}}=\frac{\beta}{\alpha} .
$$

Taking into account the above production function this yields under the assumption of positive investments

$$
\begin{aligned}
\tilde{\tilde{K}}_{i, t} & =\frac{\left(\beta w_{t}^{e}\right)^{\alpha} \tilde{Q}_{i, t}}{\left(\alpha p^{i n v}\right)^{\alpha} \min \left[A_{i, t}, B_{i, t}\right]} \\
\tilde{\tilde{L}}_{i, t} & =\frac{\left(\alpha p^{i n v}\right)^{\beta} \tilde{Q}_{i, t}}{\left(\beta w_{t}^{e}\right)^{\beta} \min \left[A_{i, t}, B_{i, t}\right]}
\end{aligned}
$$


and if $\tilde{\tilde{K}}_{i, t} \geq(1-\delta) K_{i, t-1}$ the desired capital and labor stocks read $\tilde{K}_{i, t}=\tilde{\tilde{K}}_{i, t}$ and $\tilde{L}_{i, t}=\tilde{\tilde{L}}_{i, t}$. Otherwise, we have

$$
\begin{aligned}
\tilde{K}_{i, t} & =(1-\delta) K_{i, t-1} \\
\tilde{L}_{i, t} & =\left(\frac{\tilde{Q}_{i, t}}{\left((1-\delta) K_{i, t-1}\right)^{\beta} \min \left[A_{i, t}, B_{i, t}\right]}\right)^{1 / \alpha} .
\end{aligned}
$$

For simplicity credit constraints are not incorporated in this version of the model .6 All desired investments can be financed.

The monthly realized profit of a consumption goods producer is the difference of sales revenues achieved in the malls during the previous period and costs as well as investments (i.e. labor costs and capital good investments) borne for production in the current period. In cases of positive profits, the firm pays dividends to its stockholders and the remaining profits, as well as losses, are entered on an account $A c c_{i, t}$. Similar to the capital goods producer, we assume that all households hold equal shares in all consumption goods producers, consequently the dividends are equally distributed to the households. In order to avoid exceeding accumulations of savings as well as excessive indebtedness, we employ a simple dividend policy that provides different dividend rates depending on the current balance of saving account $A c c_{i, t}$. The rule states that a firm pays no dividends, if the balance is negative and the debt is on a scale above the last monthly revenue. If the balance is positive and savings are above the monthly revenue, the firm disburses all profits. In the remaining case, if the balance is between these critical levels, a fixed proportion div $\in[0,1]$ of profits is paid out.

Since there are no constraints on the credit market and there is infinite supply of the investment good, the consumption goods producers are never rationed on the investment goods market. Wages for the full month are paid to all workers at the day when the firm updates its labor force. Investment goods are paid at the day when they are delivered.

\subsubsection{Pricing}

Consumption good producers employ a standard approach from the management literature, the so-called 'break-even analysis' (see Nagle (1987)), to set their prices. The break-even formula determines at what point the change in sales becomes large enough to make a price reduction profitable and at what point the decrease in sales becomes small enough to justify a rise in

\footnotetext{
${ }^{6}$ In contrast, in the fully fledged EURACE platform, there is an explicit credit market model which can be appropriately linked to the real sectors considered here.
} 
the price. Basically, this managerial pricing rule corresponds to standard elasticity based pricing.

Assuming that all firms have constant expectations $\varepsilon_{i}^{e}<-1$ of the elasticity of their demand, they set the price according to the standard rule

$$
p_{i, t}=\frac{\bar{c}_{i, t-1}}{1+1 / \varepsilon_{i}^{e}},
$$

where $\bar{c}_{i, t-1}$ denotes unit costs in production of firm $i$ in the previous period. Once the firm has determined the updated prices $p_{i, r, t}$ for all regions $r$ where it offers its goods, the new prices are sent to the regional malls and posted there for the following period.

\subsection{Households' consumption}

Once a month households receive their income. Depending on the available cash, that is the current income from factor markets (i.e. labor income and dividends distributed by capital and consumption goods producers) plus assets carried over from the previous period, the household sets the budget which it will spend for consumption and consequently determines the remaining part which is saved. On a weekly basis, sampling prices at the (regional) mall the consumer decides which goods to buy.

\subsubsection{The savings decision}

Our decision rule for determining the savings is based on the work of Deaton (1991). Deaton examines the saving behavior of impatient consumers when they are not permitted to borrow. In a scenario with independent and identically distributed income draws, he obtains a consumption function depending on cash on hand, which has the following characteristics: There exists a critical value of cash on hand. When the available liquidity is below this critical value the whole cash on hand will be spent. In the opposite case the agent will save a part of his cash on hand $\sqrt{7}$ The assets act like a buffer stock which protect consumption against bad income draws.

We assume a stepwise linear approximation of the consumption rule derived by Deaton $(1991,1992)$. At the beginning of period $t$, a consumer $k$ decides about the budget $B_{k, t}^{\text {cons }}$ that he will spend. In period $t$ the agent receives an income $I n c_{k, t}$, and holds assets $A s s_{k, t}$. Thus, cash on hand is denoted by $L i q_{k, t}^{\text {Avail }}=A s s_{k, t}+I n c_{k, t}$. The assets evolve according to

$$
A s s_{k, t}=L i q_{k, t-1}^{\text {Avail }}-B_{k, t-1}^{\text {cons }} .
$$

\footnotetext{
${ }^{7}$ In a more elaborate version savings will also be made dependent on the uncertainty over income.
} 
Note, that while we do not establish interest rates here, in the fully fledged EURACE framework where financial and credit markets are included, interest rates will be incorporated and become endogenous.

The consumer sets his consumption according to the following consumption rule

$$
B_{k, t}^{\text {cons }}= \begin{cases}L i q_{k, t}^{\text {Avail }}-\kappa\left(L i q_{k, t}^{\text {Avail }}-\Phi \cdot \operatorname{Inc} c_{k, t}^{\text {Mean }}\right) & \text { for } L i q_{k, t}^{\text {Avail }}>\Phi \cdot \operatorname{Inc} c_{k, t}^{\text {Mean }} \\ L i q_{k, t}^{\text {Avail }} & \text { else },\end{cases}
$$

where $\Phi \leq 1$ is a parameter, and $I n c_{k, t}^{M e a n}$ is the mean individual (labor) income of an agent over the last $T$ periods. By definition the saving propensity fulfills $0<\kappa<1$.

The implications of this consumption rule are as follows: if an agent has a current cash on hand that is below the fraction $\Phi$ of mean income, he spends all available liquidity and nothing is saved. If cash on hand exceeds $\Phi \cdot I n c_{k, t}^{M e a n}$, the agent saves a fixed fraction in order to build up a buffer stock for bad times.

The part of cash at hand that is not saved is used as the consumption budget for that month. Each consumer goes shopping once every week, so the monthly budget is equally split over the four weeks. Parts of the weekly budget that are not spent in a given week are rolled over to the consumption budget of the following week. This yields a consumption budget $B_{k, \text { week }}^{\text {cons }}$ for each week in period $t$.

\subsubsection{Selection of consumption goods}

The consumer collects information about the range of goods provided. He receives information about prices and inventories. In the Marketing literature it is standard to describe individual consumption decisions using logit models. These models represent the stochastic influence of factors not explicitly modelled on consumption decisions and the power of these models to explain real market data has been well documented (see e.g. Guadagni and Little (1983)). Therefore, we rely on a model of that kind here. We assume that a consumer's decision which good to buy is random, where purchasing probabilities are based on the values he attaches to the different choices he is aware of. Denote by $G_{k, \text { week }}$ the set of producers whose goods consumer $k$ has sampled in week week $k_{t}$ of period $t$ and where a positive stock is available at the attended mall. Since in our setup there are no quality differences between consumer goods and we also do not explicitly take account of horizontal product differentiation, choice probabilities depend solely on prices. The value of consumption good $i \in G_{k, w_{e e k}}$ is then simply given by

$$
v_{k}\left(p_{i, t}\right)=-\ln \left(p_{i, t}\right) \text {. }
$$


The consumer selects one good $i \in G_{k, w_{e e k}}$, where the selection probability for $i$ reads

$$
\operatorname{Prob}_{k, i, t}=\frac{\operatorname{Exp}\left[\lambda_{k}^{\text {cons }} v_{k}\left(p_{i, t}\right)\right]}{\sum_{i^{\prime} \in G_{k, \text { wee } k_{t}}} \operatorname{Exp}\left[\lambda_{k}^{\text {cons }} v_{k}\left(p_{i^{\prime}, t}\right)\right]} .
$$

Thus, consumers prefer cheaper products and the intensity of competition in the market is parameterized by $\lambda_{k}^{\text {cons }}$. Once the consumer has selected a good he spends his entire budget $B_{k, w e e k_{t}}^{\text {cons }}$ for that good if the stock at the mall is sufficiently large. In case the consumer cannot spend all his budget on the product selected first, he spends as much as possible, removes that product from the list $G_{k, \text { weekt }}$, updates the logit values and selects another product to spend the remaining consumption budget there. If he is rationed again, he spends as much as possible on the second selected product, rolls over the remaining budget to the following week and finishes the visit to the mall.

\subsection{Labor market}

\subsubsection{Labor demand}

Labor demand is determined in the consumption goods market. If the firms plan to extend the production they post vacancies and corresponding wage offers. The wage offer $w_{i, t}^{O}$ keeps unchanged as long as the firm can fill its vacancies, otherwise the firm updates the wage offer by a parameterized fraction. In case of downsizing the incumbent workforce, the firm dismisses workers with lowest general skill levels first.

\subsubsection{Labor supply}

Job seekers consist of a randomly determined fraction $\phi$ of employed workers who search on-the-job and the unemployed. A worker $k$ only takes the posted wage offer into consideration and compares it with his reservation wage $w_{k, t}^{R}$. A worker will not apply at a firm that makes a wage offer which is lower than his reservation wage. The level of the reservation wage is determined by the current wage if the worker is employed, and in case of an unemployed by his adjusted past wage. That is an unemployed worker will reduce his reservation wage with the duration of unemployment. When a worker applies he sends information about his general as well as his specific skill level to the firm.

\subsubsection{Matching algorithm}

According to the procedures described in the previous sections consumption goods producers review once a month whether to post vacancies for pro- 
duction workers. Job seekers check for vacancies. The matching between vacancies and job seekers works in the following way:

Step 1: The firms post vacancies including wage offers.

Step 2: Every job seeker extracts from the list of vacancies those postings to which he fits in terms of his reservation wage. Job seekers rank the suitable vacancies. The vacancy which offers the highest wage is ranked on position one and so on. If the wage offers that come with the posting are equal, vacancies are ranked by chance.

Step 3: Every firm ranks the applicants. Applicants with higher general skill $b^{g e n}$ levels are ranked higher. If there are two or more applicants with equal general skill levels, but different specific skill levels, the applicant with the higher specific skill level is ranked higher. Based on their ranking firms send job offers to as many applicants as they have vacancies to fill.

Step 4: Each worker ranks the incoming job offers according to the wages net of commuting costs (comm > 0) that may arise if he was to accept a job in the region where he does not live. Each worker accepts the highest ranked job offer at the advertised wage rate. After acceptance a worker refuses all other job offers and outstanding applications.

Step 5: Vacancies' lists and applications' lists are adjusted for filled jobs. If a firm received refusals, these applicants are dropped from the list of applicants. If all vacancies of a firm have been filled the firm refuses the other applicants and the algorithm for this firm ends.

Step 6: If the number of vacancies not filled exceeds some threshold $\bar{v}>0$ the firm raises the wage offer by a fraction $\varphi_{i}$ such that $w_{i, t+1}^{O}=\left(1+\varphi_{i}\right) w_{i, t}^{O}$. If an unemployed job seeker did not find a job he reduces his reservation wage by a fraction $\psi_{k}$, that is $\left(w_{k, t+1}^{R}=\left(1-\psi_{k}\right) w_{k, t}^{R}\right)$. There exists a lower bound to the reservation wage $w_{\text {min }}^{R}$ which may be a function of unemployment benefits, opportunities for black market activity or the value of leisure. If a worker finds a job then his new reservation wage is the actual wage, i.e. $w_{k, t}^{R}=w_{i, t}$. Go to step 1 .

This cycle is aborted after two iterations even if not all firms may have satisfied their demand for labor. As indicated above this might lead to rationing of firms on the labor market and therefore to deviations of actual output quantities from the planned quantities. In such a case the quantities delivered by the consumption good producer to the malls it serves is reduced 


\begin{tabular}{|c|c|c|c|c|c|}
\hline & \multicolumn{5}{|c|}{ General Skill Level } \\
\hline Region & 1 & 2 & 3 & 4 & 5 \\
\hline Low Skill & 0.8 & 0.05 & 0.05 & 0.05 & 0.05 \\
\hline Medium Skill & 0.05 & 0.05 & 0.8 & 0.05 & 0.05 \\
\hline High Skill & 0.05 & 0.05 & 0.05 & 0.05 & 0.8 \\
\hline
\end{tabular}

Table 2: General skill distributions in the three different types of regions.

proportionally. This results in lower stock levels and therefore increases the expected planned production quantities in the following period.

\section{$3 \quad$ Simulation results and policy experiments}

\subsection{The base case}

Before we illustrate the potential of our model for carrying out policy experiments with respect to the spatial distribution of policy measures we show that it generates time series of key economic variables with very plausible features. To that end we consider a base scenario which we will refer to as the uniform low skill scenario. Throughout the paper we assume that there are $b_{\text {max }}^{\text {gen }}=5$ levels of general skills. The function $\chi\left(b_{w}^{g e n}\right)$, which governs the speed of specific skill improvement, is chosen such that the time workers with general skill 3 need to cut the gap between their specific skill and the firm's technology level in half is the mean of the corresponding time needed by a skill level 1 and a skill level 5 worker. An analogous linear relationship also determines the adjustment speed of workers with general skill levels 2 and 4 . In a low skill region the skill distribution is such that $80 \%$ of workers have the lowest general skill level, whereas the remaining workers are equally distributed across the other four levels of general skills. Analogously, a region is a medium skill or high skill regions if $80 \%$ of workers have general skill level 3 respectively 5 . We summarize the skill distributions in three types of regions in table 2. Although none of these distributions match empirical skill distributions in industrialized countries we still use them to show the qualitative effects of policies influencing the skill distribution.

In the uniform low skill scenario both regions are low skill regions. In addition we assume that there is a $10 \%$ probability of a quality improving innovation in the investment goods sector per month and each innovation on average increases the quality of the investment good by $5 \%$. This corresponds to an average productivity growth rate of $6 \%$ if specific skills were 
sufficiently large to fully exploit all new technologies. Simulations are run for 250 months which corresponds to about 20 years. The parameter values have been calibrated using empirical evidence from different streams of relevant literature. In table 4 in the Appendix the used parameter values are summarized and we also briefly discuss the sources for the estimates. In order to study the implications if the factor labor is much less mobile than capital and consumption goods we consider relatively high commuting costs that make commuting to work across regions rather exceptional.

In figure 1 we show the time series for a single run of output, unemployment, (skill-dependent) wages and specific skills along the technology frontier in a typical run in the uniform low-skill scenario.

Output is increasing over time with some fluctuations, where growth rates oscillate and their temporary mean seems to be closely connected to the development of the quality of the capital stock and the level of specific skills. These two levels grow slowly in the beginning of the run but increase substantially after period $t=150$ and it can be clearly seen that this induces growth in output and wages as well as a reduction in unemployment after $t=150$. The relatively low growth rates and relatively high unemployment therefore seems to be an implication of the low general skills in the considered regions. Wages and the rate of unemployment differ between the different skill groups, where the dominant group of low-skill workers faces a higher rate of unemployment (not shown in panel). Wages of the workers with the highest skills are higher than the wages of workers with the lowest skills. Productivity improving innovations shift the technological frontier. Average quality of the capital stock grows as well but is by construction below the technological frontier. Average specific skills increase also over time but constitute the limiting factor for exploiting the technological possibilities of the capital employed in the production process. This brief discussion illustrates that the model exhibits qualitative effects that are very plausible within the framework considered in our setup. Skill level differences have an impact on the individual level with respect to wages and unemployment that are well in accordance with empirical observations. Furthermore, it seems that in principle technological change that improves investment good productivity might have positive or negative effects on aggregate employment depending on the short-term rate of technological change.

\section{$3.2 \quad$ Policy experiments}

The framework described here allows for a number of policy experiments that seem to be of high relevance for policy makers concerned with strengthening economic growth and employment in the considered regions. As discussed 

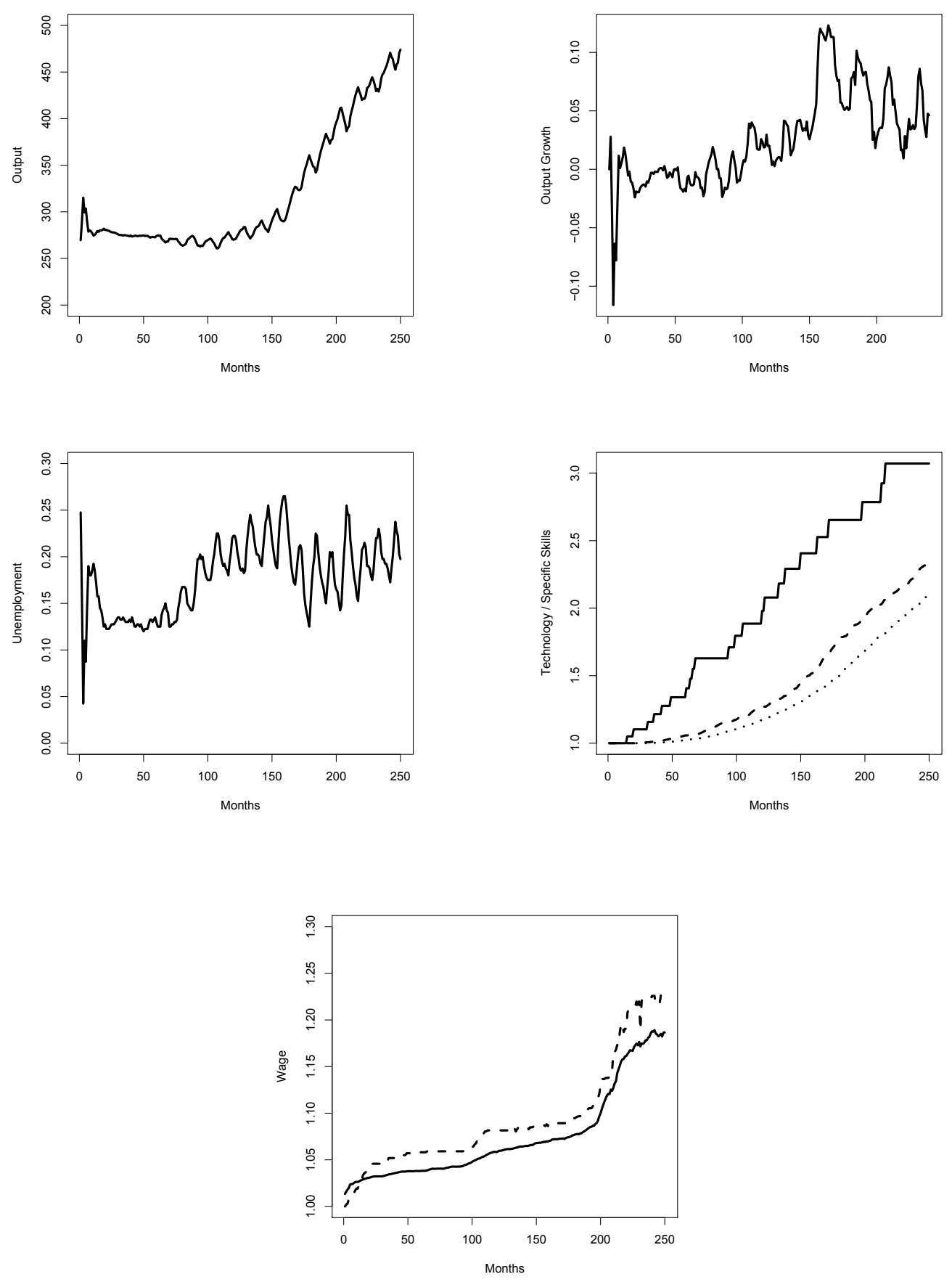

Figure 1: Single runs for the uniform low skill scenario; starting from the upper left panel: output; output growth; unemployment rate; technology frontier (solid line), average quality of capital (dashed line) and average specific skills (dash-dotted line); average wages of workers with lowest (solid line) and highest (dashed line) generæ20 skill level; 
in the introduction, generally speaking the model allows to evaluate policies influencing skill distributions among workers, policies influencing the speed at which the technological frontier develops and, what might be most interesting and innovative, also the interplay of policies with these two objectives.

The version of our framework presented here captures the effects of policies mentioned in the introduction (funding of basic and applied research, financial incentives for R\&D efforts by firms, providing infrastructure and incentives for $\mathrm{R} \& \mathrm{D}$ cooperation and intensive knowledge exchange) in reduced form through the parameters $\gamma^{i n v}$ and $\Delta q^{i n v}$. Policy efforts aiming at a speed-up of the movement of the technological frontier should lead to an increase in $\gamma^{i n v}$, whereas attempts to foster basic research and to move from incremental to more fundamental innovations in the investment goods sector should trigger an increase in $\Delta q^{i n v}$. Our framework allows to examine how such policies would influence the relative performance of different policies that influence the skill distribution. Understanding such cross-effects is essential to coordinate the different policy measures and to design a well balanced package of measures where the negative interaction terms between the different parts are avoided.

We illustrate the potential insights from such policy experiments by considering a simple example. Starting from the uniform low skill scenario we assume that a policy-maker responsible for economic development in both regions considers to launch a campaign in order to increase the general skill level of workers in the region. Such a campaign might involve improvements in the system of lower and higher education, providing incentives for firms to train workers, e-learning activities and so on. Furthermore, it is assumed that using the budget assigned to this campaign the policy-maker can either improve both regions from low-skill to medium-skill regions or, if all efforts are invested in only one region, improve one region to a high-skill region with the other region remaining low-skill. Since in both cases the total number of general-skill units added to the work force is identical, it is a-priori a nontrivial question whether the two policy options yield identical results and, if not, in which way the effects will differ.

In order to address this question we have run batches of 50 simulation runs for the uniform medium and low-high scenarios and compare them with each other and with the base case of uniform low-skill regions. In figure 2 we depict mean trajectories over the 50 runs in the three scenarios. As can be seen, the difference in the effect of the two policy measures is quite striking. Focussing the resources to improve the general skill level of workers in one region only (low/high scenario) yields relatively high output in the medium run but worse results in terms of long run output compared to both the uniform medium and the uniform low skill scenario. Raising the general skill level of workers 


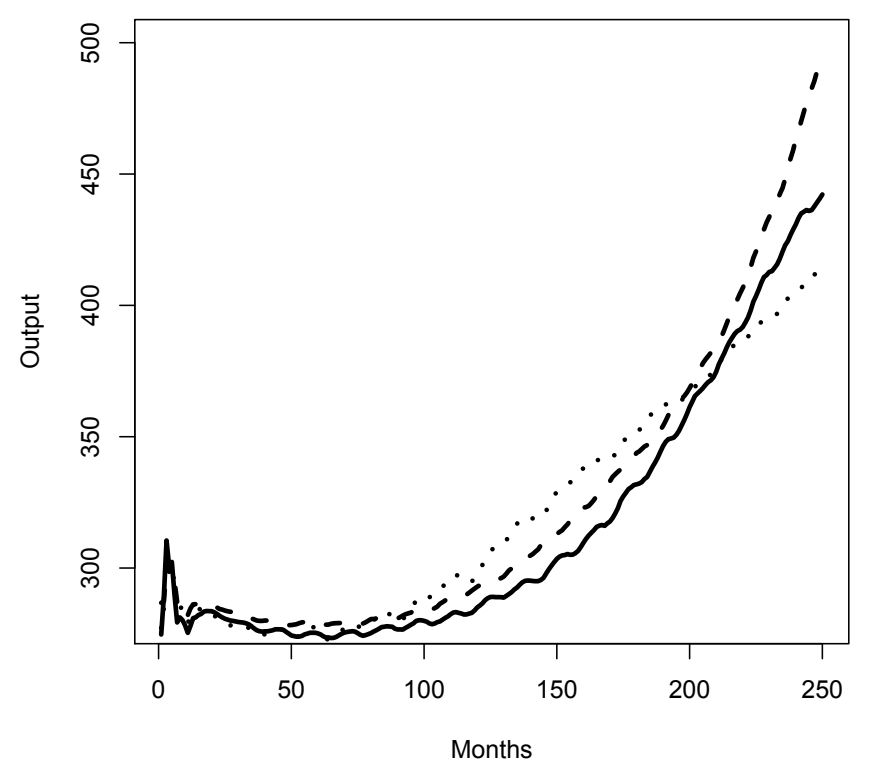

Figure 2: Batch run for outputs in uniform low scenario (solid line), uniform medium scenario (dashed line), and low/high scenario (dotted line)

in both regions (uniform medium) yields an output that is always higher than in the uniform low skill case. The large impact of the spatial allocation of policy measures in this simple example highlights the strongly non-linear and path dependent nature of a micro-founded agent-based model which leads to non-trivial relationships between policy intervention and emergent results. To show that the observations made in figure 2 are representative of the whole batch of simulation runs we have carried out a statistical analysis by applying a Wilcoxon test for equalities of means to output data in the two scenarios at $t=150$ and $t=250$. The results are given in table 3 and we conclude that the observed effects of the different policies are indeed statistically significant at a level of $93 \%$.

A more detailed consideration of the mechanisms underlying the different effects of these policy measures shows that the interplay of wage-dynamics, wage-driven price dynamics, and barriers to skill transfer through commuting workers that arises in the high-low scenario is responsible for the larger growth of output and employment in the uniform medium scenario.

In the low/high scenario depicted in the upper left panel of figure 3 the average specific skills increase more in the region at which the policy measure 


\begin{tabular}{|c|c|c|c|}
\hline & uniform medium & & low/high \\
\hline $\mathrm{t}=150$ & 312.6 & $\underset{(0.0648)}{<}$ & 329 \\
\hline $\mathrm{t}=250$ & 496.8 & $\underset{(0.0637)}{>}$ & 415.5 \\
\hline
\end{tabular}

Table 3: Average output over 50 runs in the two scenarios and the p-values of the Wilcoxon test for equality of means.
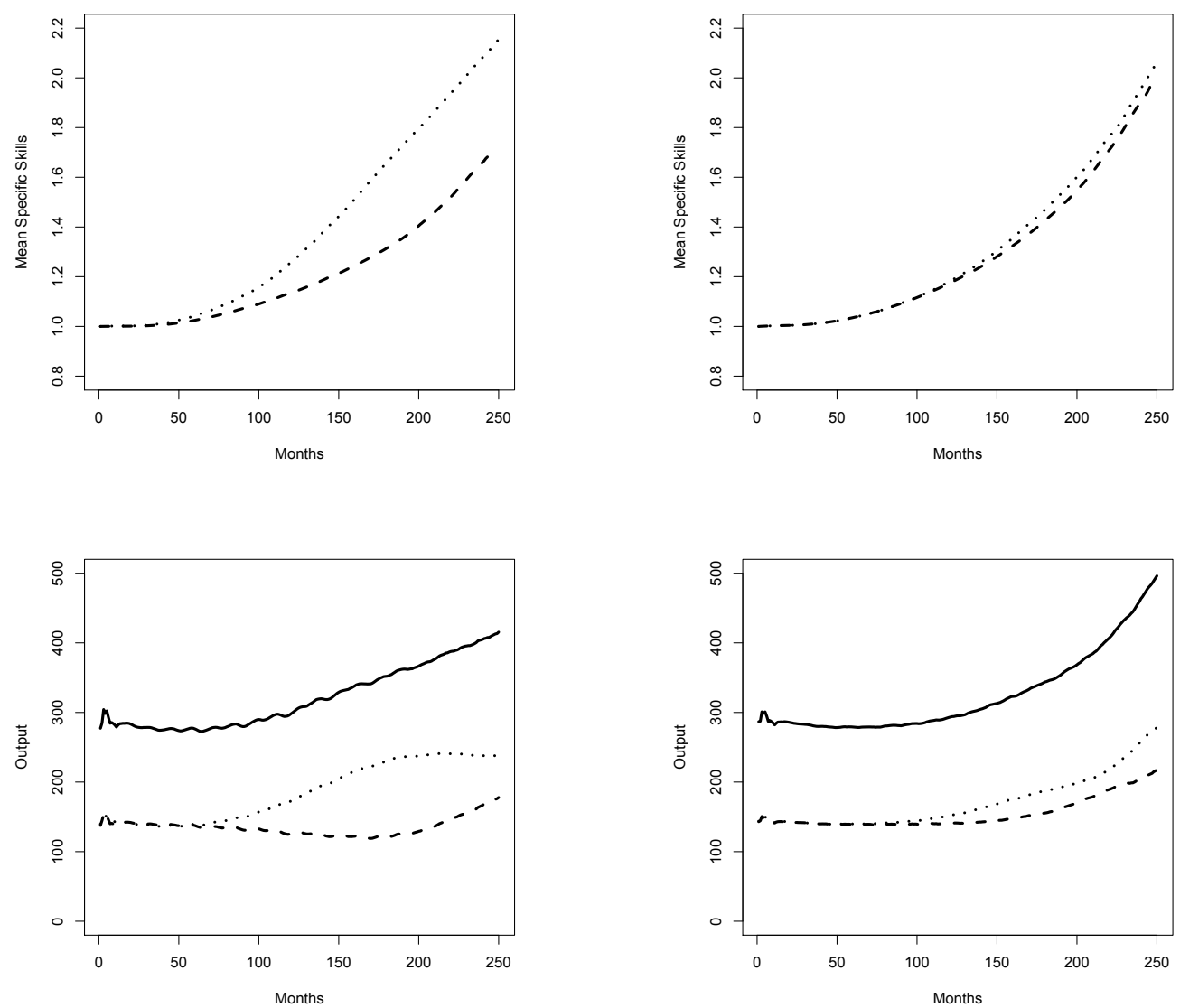

Figure 3: Batch runs for the low/high scenario (left column) and the uniform medium scenario (right column); panels in first row show specific skills in both regions, panels in second row show total output (solid line) and output in both regions; 
is targeted. Obviously, workers learn the specific skills more quickly as their general skills are higher compared to the low/low benchmark, while no such process arises in the region without improvements in the general skills of the workers. Allocating resources to improve general skills equally across regions yields the expected results that the improvement of average specific skills is about equal in both regions (upper right panel). The lower two panels give insight into the workings of the model by showing the diverging patterns of output across the two regions comparing the two policies. In the low/high scenario output increases for the region with the higher average specific skill level. Clearly, firms in this region take advantage of the more skilled workforce which allows them to exploit the technological improvement of their capital stock to a larger degree than the firms in the other region. It seems, however, that this increase in output at least partly arises at the expense of the low skill region where up to $t=200$ we actually observe a decline in output over time. Adding up regional outputs yields an increasing total output, but total output does not increase so strongly as in the uniform medium skill scenario depicted in the lower right panel. Here, as average specific skills in both regions have increased somehow due to the equal distribution of policy efforts, output increased also in both regions. In total this has a larger impact on output in the longer run which explains why targeting the policies to only one region turns out to be the inferior policy with respect to output gains.

Looking at prices and commuting employees in the low/high scenario corroborates the analysis of our major finding of the policy experiment. Due to the faster adaptation of specific worker skills to the improving technological frontier firms in the high-skill region after a short initial phase produce with lower unit costs compared to those in the low skill region. It should be noted that this observation holds true in spite of the fact that wages in the high skill region are higher than those in the low skill region (not shown in our figures). As can be seen in figure 4 in the left panel the cost advantage translates to a lower price of the goods offered by producers from the highskill region and this shifts demand away from producers in the low skill region towards producers in the high skill region. However producers in the high skill regions are limited in their output expansion by the lack of additional local workers. They are not able to hire workers from the low skill region upon the increase in demand because this is associated with labor market frictions and commuting costs for the workers. This implies an upper bound to output expansion for producers in the high skill region and accelerated upwards trend for wages. Therefore, the increase in output in the high skill region is not as fast as it could be based on the level of specific skills and, as can be seen in the lower left panel of figure 3, eventually comes to a hold. 

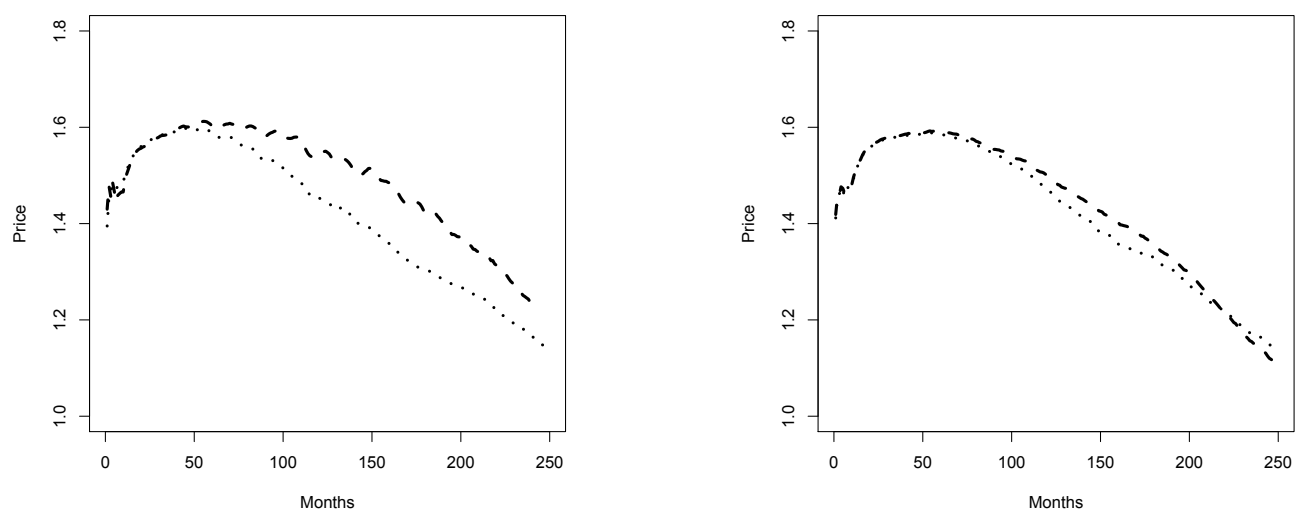

Figure 4: Batch runs mean prices of producers located in the two regions. Left panel shows high skill (dotted line) and the low skill (dashed line) region for the low/high scenario; right panel shows the uniform medium scenario.

This economic mechanism, which drives our results, can be more clearly observed by considering single runs than averages, where, due the fact that the timing of the expansion and rationing phases differ quite a bit between runs, the link between the lack of mobility of labor, prices and output is less obvious. In figure 5 prices, unemployment and output in the high and in the low-skill region is shown for a single run. As has to be expected initially producers in the high-skill region gain a cost advantage and are able to charge a lower price. The resulting shift in demand towards producers from the highskill region reduces unemployment in that region to almost zero but at the same time increases unemployment in the low skill region. The rationing of producers in the high-skill region on the labor market has two implications. First, these producers cannot fully serve the demand for their products and, second, the price gap between the two products goes down. Both effects limit the increase in output of the producers of the high-skill region. Overall total output grows at a lower rate than it would if general skill levels were uniform across regions. In this particular run the producers in the high-skill region are rationed on the labor market rather early and the induced wage increase in that region leads to a price advantage of producers in the low-skill region after about period $t=110$. As can be seen in figure 4 on average the reduction of the price gap is much slower than in this particular example. To give a general economic interpretation of the observed phenomenon, we conclude that the relatively low mobility of the factor labor compared to that of consumption goods is responsible for the incomplete substitution of 
production in the low skill region with production in the high skill region. Furthermore, the falling demand for goods of producers in the low skill region and commuting costs prevents them from hiring high-skill workers from the other region and therefore no technological spillovers from the high to the low skill region emerge. Together these effects make the geographically focused policy less effective than one leading to a uniform increase of general skills in all regions.
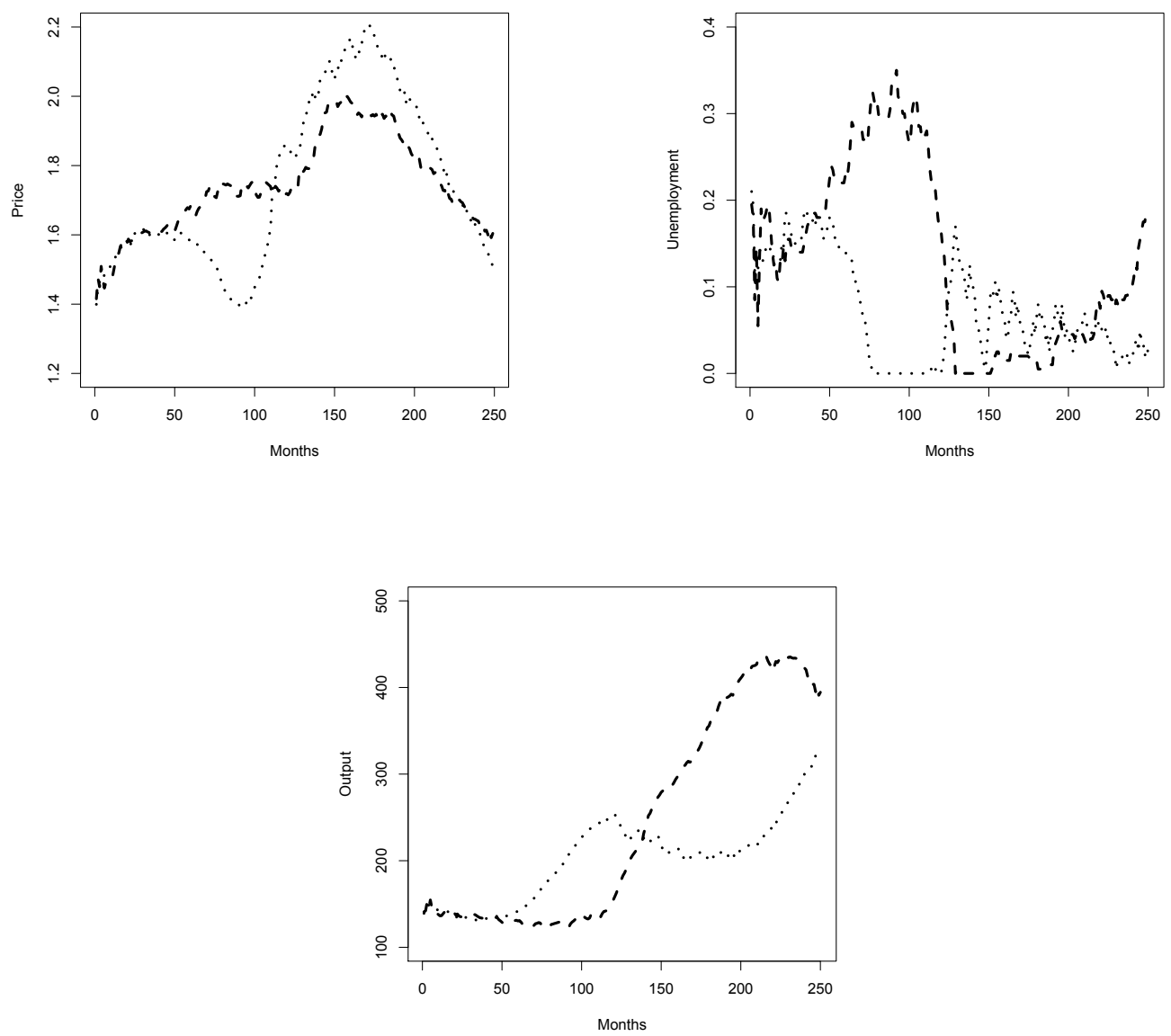

Figure 5: A single run for the low/high scenario: prices (upper left panel), unemployment (upper right panel) and output (lower panel) in the high-skill (dotted line) and the low-skill (dashed line) region. 


\section{Conclusions}

Agent-based models are not only a useful tool to transcend borders set by standard analytical models for the study of economic systems but also open up promising avenues for economic policy design. We developed a still parsimonious micro-based macroeconomic model to study the role of innovation and skills for economic growth and employment in a regional setting. Our policy experiments - which as we believe are of high relevance for policymakers concerned about the right mix of policies targeted toward improving specific or general skills, and giving firms incentives to innovate - yield very plausible insights, highlighting the role of non-trivial interaction effects in a dynamic setting which are hard to analyze in more standard static frameworks. Without stressing the results of our policy experiments too strongly there occurs to be a point in allocating resources to improve general skills equally across regions to improve output in the long run.

The policy experiments presented in this paper clearly illustrate the more general point that the ability of agent-based models to capture regional and individual heterogeneity, market imperfections and dynamic effects on different scales makes them a powerful tool for policy advice. These type of models cannot only produce meaningful qualitative insights, but, if carefully calibrated, one can also expect quantitatively informative guidance for policy makers. The increasing availability of computing power allows to run agent-based models that, on the one hand, capture the fact that economic interactions on the micro level are extremely numerous, and, on the other hand, incorporate institutional details that seem important for concrete policy advice.

On the list of extensions to the model the endogenization of technological change and the explicit introduction of fiscal policy loom high. Finally, as pointed out earlier, this project is part of a larger endeavor. Within EURACE we aim at integrating models for the credit and financial markets build by our partners to finally arrive at a fully fledged closed macroeconomic model. Obviously, this will add many more possibilities to conduct policy analyses of the type we were trying to promote in our attempt here.

\section{Acknowledgements}

This research was funded by the European Commission as part of the FP6STREP project EURACE ('An agent-based software platform for European economic policy design with heterogeneous interacting agents: new insights from a bottom up approach to economic modeling and simulation'). The 
aim of the research project is to build a software platform for evaluating European economic policies. We are thankful to our colleagues for making valuable contributions to this part of the research project. The model has been implemented in FLAME, an agent-based simulation environment developed by project partners working in the area of software engineering. A list of project participants and further information on EURACE is available at www.eurace.org. We are grateful for helpful comments on this paper from three referees and Peter Winker. 


\section{Appendix}

Table 4: Parameter settings

\begin{tabular}{|c|c|c|}
\hline Description & Parameter & Value \\
\hline Number of households: & & 400 \\
Number of firms & & 10 \\
Number of regions & $R$ & 2 \\
Labor Intensity of Production & $\alpha$ & 0.662 \\
Capital Intensity of Production & $\beta$ & 0.338 \\
Innovation probability & $\gamma^{i n v}$ & 0.1 \\
Depreciation rate of capital & $\delta$ & 0.01 \\
Monthly Discount factor & $\rho$ & 0.95 \\
Mark-up factor & 1 & 0.2 \\
Wage update & $\varphi_{i}^{e} \mid-1$ & 0.02 \\
Reservation wage update & $\psi_{k}$ & 0.02 \\
Minimal reservation wage & $w_{\text {min }}^{R}$ & 1 \\
Marginal saving propensity & $\kappa$ & 0.1 \\
Intensity of choice by consumers & $\lambda_{k}^{c o n s}$ & 8.5 \\
Commuting costs & comm & 1 \\
Fraction of on-the-job searchers & $\phi$ & 0.1 \\
\hline \multicolumn{2}{|c}{} \\
\hline \multicolumn{2}{|c}{} \\
\hline
\end{tabular}

The simulation results of our model are based on a deliberate calibration of the model. Whenever possible the parameters, as summarized in table 4, were chosen to reflect empirical evidence. The ratio of the number of households (workers) and firms that we implemented matches mean firm sizes to be observed in Europe. ${ }^{8}$ Estimates of labor intensity of the German Statistical office, see Bundesamt (2004), suggest $\alpha=0.662$ so that we have $\beta=0.338$ given our assumption of a constant returns to scale production function. The innovation probability $\gamma^{i n v}$ was chosen to reflect estimates approximating shifts of the technological frontier. Comparable to data reported in Aghion et al. (2006) our calibration yields a growth rate of the technological frontier of around $6 \%$ per year if skills were sufficient to fully exploit technological innovations. The calibration of the yearly depreciation rate follows what is reported in Bundesamt (2006). Our choice for the markup is based on the empirical evidence reported in Small (1997). We take the estimate for motor cycle production as a guideline for a markup of 20 percent. Wage updates $\left(\varphi_{i}\right)$ are calibrated to match wage growth in Germany

\footnotetext{
${ }^{8}$ See http://epp.eurostat.eu.europa.eu.
} 
during the decade of full employment in the sixties.9 ${ }^{9}$ The parameter value for the adjustment of the reservation wage $\left(\psi_{k}\right)$ was chosen based on reported wage losses of approximately $17 \%$ after spells of unemployment in Germany (see Burda and Mertens (2001)), and an average duration of unemployment of 30 weeks which matches German data. As a proxy for the reservation wage we make use of the net replacement rates of unemployment benefit schemes in OECD countries (OECD (2004)). For the marginal propensity to save we chose $\kappa=0.1$, which is close to the savings rate in Germany in previous years. The calibrated value for the intensity of the consumer choice stems from estimated multinomial logit models of brand selection. Estimates based on market data by, e.g. Krishnamruthi and Raj (1988), provide a lower bound for $\lambda_{k}^{c o n s}$, which captures choices between brands that are available in the same local mall. These considerations suggest the value of $\lambda_{k}^{\text {cons }}=8.5$ which we have chosen. Finally, we let $10 \%$ of the employed search on-the-job which is in the range of ratios reported in Rosenfeld (1977), Black (1981), or Pissarides and Wadsworth (1994).

\section{References}

Aghion, P. (2007). Growth and the financing and governance of education. Keynote Lecture for the 2007 Meeting of the German Economic Association.

Aghion, P., Blundell, R., Griffith, R., Howitt, P., and Prantl, S. (2006). The effects of entry on incumbent innovation and productivity. NBER Working Paper No. 12027.

Argote, L. and Epple, D. (1990). Learning curves in manufacturing. Science, 247:920-924.

Audretsch, D. and Feldman, M. (2004). Knowledge spillovers and the geography of innovation. In Henderson, J. and Thisse, J.-F., editors, Handbook of Regional and Urban Economics Volume 4: Cities and Geography, pages 2715-2739. North-Holland.

Bassanini, A. (2004). Improving skills for more and better jobs? the quest for efficient policies to promote adult education and training. European Economy: Special Reports, 4(3):103-137.

\footnotetext{
${ }^{9}$ See, for instance, www.sachverstaendigenrat-wirtschaft.de/timerow/tabdeu.php.
} 
Bassanini, A. and Scarpetta, S. (2002). Does human capital matter for growth in oecd countries? a pooled mean group approach. Economics Letters, 74(3):399-405.

Black, M. (1981). An empirical test of the theory of on-the-job-search. The Journal of Human Resources, 16:129-141.

Bosker, M., Brakman, S., Garresten, H., and Schramm, M. (2007). Adding geography to the new economic geography. Working Paper No. 2038, Cesifo.

Bundesamt, S. (2004). Volkswirtschaftliche Gesamtrechnung.

Bundesamt, S. (2006). Fachserie 18, 1.4 Inlandsproduktberechnung - Detaillierte Jahresergebnisse 2006.

Bunn, D. W. and Oliveira, F. (2001). An application of agent-based simulation to the new electricity trading arrangements of england and wales. IEEE Transactions on Evolutionary Computation, 5(5):493-503.

Burda, M. and Mertens, A. (2001). Estimating wage losses of displaced workers in germany. Labour Economics, 8:15-42.

Chiaromonte, F. and Dosi, G. (1993). Heterogeneity, competition and macroeconomic dynamics. Structural Change and Economic Dynamics, 4:39-63.

Cyert, R. M. and March, J. G. (1963). A Behavioral Theory of the Firm. Prentice Hall, Englewood Cliffs.

Dawid, H. and Fagiolo, G. (2007). Agent based models for economic policy design. Forthcoming special issue of the Journal of Economic Behavior and Organization.

Dawid, H. and Wersching, K. (2006). On technological specialization in industrial clusters: An agent-based analysis. In Rennard, J.-P., editor, Handbook of Research on Nature Inspired Computing for Economics and Management, pages 367-378. Idea.

Deaton, A. (1991). Saving and liquidity constraints. Econometrica, 59:12211248.

Deaton, A. (1992). Houshold saving in ldcs: Credit markets, insurance and welfare. Scandinavian Journal of Economics, 94:253-273. 
Delli Gatti, D., Guilmi, C., Gaffeo, E., Giulioni, G., Gallegati, M., and Palestrini, A. (2005). A new approach to business fluctuations: Heterogeneous interacting agents, scaling laws and financial fragility. Journal of Economic Behavior and Organization, 56:489-512.

Döring, T. and Schnellenbach, J. (2006). What do we know about geographical knowledge spillovers and regional growth?: A survey of the literature. Regional Studies, 40(3):375-395.

Dosi, G., Fagiolo, G., and Roventini, A. (2006). An evolutionary model of endogeneous business cycles. Computational Economics, 27(1):3-34.

Fagiolo, G., Dosi, G., and Gabriele, R. (2004). Matching, bargaining, and wage setting in an evolutionary model of labor market and output dynamics. Advances in Comples Systems, 14:237-273.

Geroski, P. (1995). Markets for technology: Knowledge, innovation and appropriability. In Stoneman, P., editor, Handbook of the Economics of Innovation and Technological Change. Blackwell.

Gintis, P. (2006). The dynamics of general equilibrium. The Economic Journal, 117:1280-1309.

Griffith, R., Redding, S., and van Reenen, J. (2004). Mapping the two faces of r\&d: Productivity growth in a panel of oecd industries. Review of Economics and Statistics, 86(4):883-895.

Guadagni, P. and Little, J. (1983). A logit model of brand choice calibrated on scanner data. Marketing Science, 2(3):203-238.

Happe, K., Balmann, A., Kellermann, K., and Sahrbacher, C. (2007). Does structure matter? the impact of wwitching the agricultural policy regime on farm structures. Journal of Economic Behavior and Organization. forthcoming.

Henderson, J. and Thisse, J.-F. E. (2004). Handbook of Regional and Urban Economics Volume 4: Cities and Geography. North-Holland.

Hillier, F. and Lieberman, G. (1986). Introduction to Operations Research. Holden-Day.

Kirman, A. (1992). Whom or what does the representative individual represent? Journal of Economic Perspectives, 6:117-136. 
Krishnamruthi, L. and Raj, S. P. (1988). A model of brand choice and purchase quantity price sensitivities. Marketing Science, 7:1-20.

Krugman, P. (1991). Increasing returns and economic geography. Journal of Political Economy, 99(3):483-499.

Malerba, F., Nelson, R., Orsenigo, L., and Winter, S. (2001). Competition and industrial policies in a 'history-friendly' model of the evolution of the computer industry. Journal of Industrial Organization, 19(5):635-664.

Mühlemann, S., Wolter, S., Fuhrer, M., and Wüest, A. (2007). Lehrlingsausbildung - ökonomisch betrachtet: Ergebnisse der zweiten Kosten-NutzenStudie. Rügger.

Nagle, T. (1987). Strategy and Tactics of Pricing. Prentice Hall.

Nelson, R. R. and Winter, S. G. (1982). An Evolutionary Theory of Economic Change. Harvard University Press, Cambridge, Mass. and London.

Neugart, M. (2007). Labor market policy evaluation with ace. Journal of Economic Behavior and Organization. forthcoming.

Nicolaisen, J., Petrov, V., and Tesfatsion, L. (2001). Market power and efficiency in computational electricity market with discriminatory double-auction pricing. IEEE Transactions on Evolutionary Computation, 5(5):504-523.

OECD (2000). Is there a new economy? First report on the oecd growth project, OECD, Paris. Internet source; http://www.oecd.org/growth.

OECD (2004). Benefits and Wages: OECD indicators.

Ottaviano, G. and Thisse, J.-F. (2004). Agglomeration and economic geography. In Henderson, J. and Thisse, J.-F., editors, Handbook of Regional and Urban Economics Volume 4: Cities and Geography, pages 2563-2608. North-Holland.

Phelps, S., Parsons, S., McBurney, P., and Sklar, E. (2002). Co-evolution of auction mechanisms and trading strategies: Towards a novel approach to microeconomic design. Proceedings of the Workshop on Evolutionary Computation in Multi-Agent Systems (ECoMAS 2002).

Pissarides, C. and Wadsworth, J. (1994). On-the-job-search, some empirical evidence. European Economic Review, 38:385-401. 
Rosenfeld, C. (1977). The extent of job search by employed workers. Monthly Labor Review, 100:39-43.

Silverberg, G. and Verspagen, B. (1993). Collective learning, innovation and growth in a boundedly rational, evolutionary world. Journal of Evolutionary Economics, 4:207-226.

Simon, H. A. (1978). Rationality as process and as product of thought. American Economic Review, 68(2):1-16.

Simon, H. A. (1983). Reasons in Human Affairs. Stanford University Press, Stanford.

Small, I. (1997). The cyclicality of mark-ups and profit margins: Some evidence for manufacturing and services. Bank of England Working Paper.

Tesfatsion, L. and Judd, K. E. (2006). Handbook of Computational Economics II: Agent-Based Computational Economics. North-Holland.

Wersching, K. (2007). Agglomeration in an innovative and differentiated industry with heterogneous knowledge spillovers. Journal of Economic Interactions and Coordination, 2(1):1-25.

Windrum, P., Fagiolo, G., and Moneta, A. (2007). Empirical validation of agent-based models: Alternatives and prospects. Journal of Artificial Societies and Social Simulation, 10(2). 